\title{
Erratum to: Neuropsychological Functioning After Implantable Cardioverter-Defibrillator Surgery
}

\author{
Abdullah Alabdulgader
}

\section{Erratum to:}

Chapter 2 in: R. Proietti et al. (eds.), Psychological, Emotional, Social and Cognitive Aspects of Implantable Cardiac Devices, https://doi.org/10.1007/978-3-319-55721-2_2

We received revised caption for figure 2.10 and the same has been updated in the chapter. The corrected figure caption is available below.

Fig. 2.10 (a, b) Computational tools for identifying PTSD biomarkers from "multiomic" data, illustrated biomarkers provide both a means for diagnosing new patients as well as a molecular network-level description of the PTSD phenotype. (a) Reproduced with permission of the Regents of The University of California (b) IEEE grants to Prof. Abdullah Alabdulgader, License Number 4177010395849, a non-exclusive, non-transferable worldwide license to use this image in accordance with the terms and conditions of the agreement 